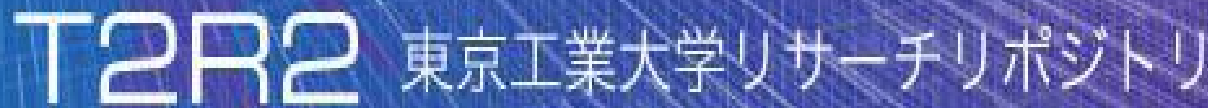

\section{Tokyo Tech Research Repository}

\section{論文 /著書情報 \\ Article /Book Information}

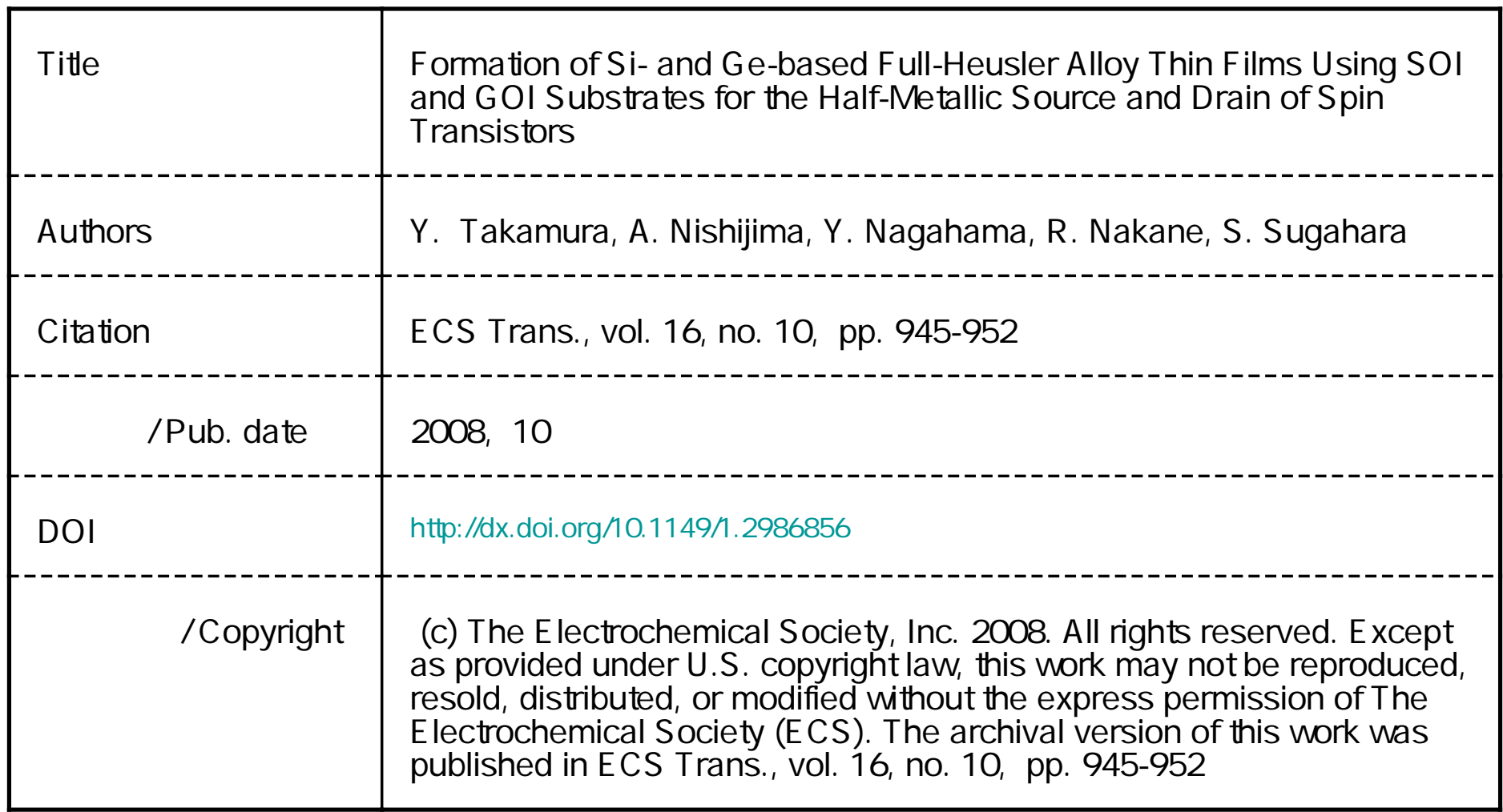




\title{
Formation of Si- and Ge-based Full-Heusler Alloy Thin Films Using SOI and GOI Substrates for the Half-Metallic Source and Drain of Spin Transistors
}

\author{
Y. Takamura ${ }^{\mathrm{a}}$, A. Nishijima ${ }^{\mathrm{a}}$, Y. Nagahama ${ }^{\mathrm{a}}$, R. Nakane ${ }^{\mathrm{b}}$, and S. Sugahara ${ }^{\mathrm{a}, \mathrm{c}}$ \\ ${ }^{a}$ Imaging Science and Engineering Laboratory, Tokyo Institute of Technology, \\ Yokohama 226-8502, JAPAN \\ ${ }^{\mathrm{b}}$ Department of Electrical Engineering and Information Systems, The University of \\ Tokyo, Tokyo 113-8656, JAPAN \\ ${ }^{c}$ CREST, Japan Science and Technology Agency, Kawaguchi, 332-0012, JAPAN
}

The paper presents a novel preparation technique for Si- and Gebased half-metallic full-Heusler alloy thin films, utilizing siliconon-insulator (SOI) and germanium-on-insulator (GOI) substrates, respectively. Full-Heusler $\mathrm{Co}_{2} \mathrm{FeSi}\left(\mathrm{Co}_{2} \mathrm{FeGe}\right)$ alloy thin films were successfully formed by thermally activated silicidation (germanidation) reaction between an ultra-thin SOI (GOI) layer and $\mathrm{Co} / \mathrm{Fe}$ layers deposited on it. This technique can easily produce fully ordered $L 2_{1}$ structure that is necessary for the halfmetallicity of full-Heusler alloys. The proposed technique is compatible with metal source/drain formation process in advanced CMOS technology and would be applicable to the fabrication of the half-metallic source/drain of MOSFET type of spin transistors.

\section{Introduction}

In recent years, spin transistors ${ }^{1-4}$ attract considerable attention, since they have interesting transistor behavior with a new degree of freedom in controlling output currents based on spin-related phenomena. This feature makes it possible that spin transistors have useful nonvolatile and reconfigurable functionalities to improve circuit performance beyond present transistors. However, high current drive capability and a large on/off current ratio are still important requirements even for spin transistors, as well as present ordinary transistors ${ }^{4}$. In addition, excellent scalability and integration ability are also indispensable to establish very large scale integration. From these points of view, one of the promising spin transistors is recently proposed spin metal-oxidesemiconductor field-effect transistors (spin MOSFETs). ${ }^{3,4}$ The basic structure of spin MOSFETs consists of a MOS capacitor and ferromagnetic contacts for the source and drain, and the device performance depends on the material of the ferromagnetic source/drain. Half-metallic ferromagnets (HMFs) are the most effective source/drain material to realize the spindependent output characteristics of spin MOSFETs, ${ }^{3,4}$ owing to their extremely high spin polarization and unique spin-dependent band structure.

Recently emerging silicide-based metal source/drain MOSFET (Ref. 6) technology would be applicable to the fabrication of spin MOSFETs, due to the similarity in the device structure between metal source/drain MOSFETs and spin MOSFETs. For metal source/drain MOSFETs, transition metal (or rare earth metal) silicides are directly used as their source and drain. These silicides are formed by thermally activated intermixing reaction, the so-called silicidation, between a Si substrate and transition metal (or rare earth metal) layer deposited on the Si substrate. In general, the thermal process for 
silicidation is induced by rapid thermal annealing (RTA). $L 2_{1}$-phase half-metallic fullHeusler alloys containing $\mathrm{Si}\left(\mathrm{Co}_{2} \mathrm{FeSi}^{7,8}, \mathrm{Co}_{2} \mathrm{MnSi}^{9}\right.$, etc. $)$ are one of attractive candidates for the HMF source/drain of spin MOSFETs, since these materials are considered to be a kind of silicides and thus they have a possibility to be formed by the RTA-induced silicidation process that is compatible with present CMOS technology.

Ge-based full-Heusler alloys, such as $\mathrm{Co}_{2} \mathrm{FeGe}^{10}$ and $\mathrm{Co}_{2} \mathrm{MnGe}^{11}$, are applicable to spin MOSFETs with the Ge channel. Recently, metal source/drain fabrication process employing RTA for Ge-channel MOSFETs ${ }^{12}$ has been emerging. Full-Heusler alloy thin films containing $\mathrm{Ge}$ would also be formed by RTA-induced metal-source/drain germanidation process, resulting in Ge-channel spin MOSFETs compatible with advanced Ge-channel CMOS technology.

In this paper, we present a RTA-induced formation technique for $L 2_{1}$-phase $\mathrm{Co}_{2} \mathrm{FeSi}^{13}$ and $\mathrm{Co}_{2} \mathrm{FeGe}$ full-Heusler alloy thin films, utilizing a silicon-on-insulator (SOI) and germanium-on-insulator (GOI) substrates. Magnetic properties in the RTA-formed $\mathrm{Co}_{2} \mathrm{FeSi}$ and $\mathrm{Co}_{2} \mathrm{FeGe}$ full-Heusler alloy films were also investigated.

\section{Proposed preparation method}

The problem of RTA technique for ferromagnetic silicides including Si-containing full-Heusler alloys is the formation of nonmagnetic silicides with low magnetic element content. This is caused by the deep diffusion of magnetic elements into a Si substrate. In other words, when the much amount of Si atoms is supplied from the Si substrate, the most thermodynamically favorable nonmagnetic silicides are preferentially formed. Figure 1 shows the proposed preparation method of full-Heusler alloys employing a SOI substrate. The diffusion of magnetic atoms is blocked by the buried oxide (BOX) layer of the SOI substrate, and thus the composition of the RTA-formed film layer can be easily controlled by the thickness of the magnetic metal and SOI layers. When the amount of constituent elements of the resulting film layer is limited (or adjusted) to the chemical composition of full-Heusler alloys, homogeneous full-Heusler alloys would be formed, since full-Heusler alloys are also one of thermodynamically stable phases as long as keeping their (near) stoichiometric composition. Recently, ferromagnetic $\mathrm{Fe}_{3} \mathrm{Si}$ that has the $L 2_{1}$-related $\mathrm{DO}_{3}$ structure was successfully formed by this technique. ${ }^{14}$ Full-Heusler alloy thin films containing Ge are also expected to be formed by the same manner using GOI substrates.

\section{Experimental procedure}

\section{$\underline{\mathrm{Co}_{2}} \underline{\mathrm{FeSi}}$}

All the experiments for $\mathrm{Co}_{2} \mathrm{FeSi}$ were carried out using commercially available wafer-bonding SOI substrates that consist of a thin SOI layer, BOX layer, and $\mathrm{Si}$ substrate (hereafter, this structure is referred to as $\mathrm{SOI} / \mathrm{BOX} / \mathrm{Si}$ ). A SOI substrate sample was cleaned by chemical oxidation using $\mathrm{H}_{2} \mathrm{SO}_{4}$ and $\mathrm{H}_{2} \mathrm{O}$ mixture and successive etching of the resulting surface oxide by buffered HF solution. Then $\mathrm{Co}$ and Fe films were deposited on the SOI layer surface in an ultrahigh vacuum. Subsequently, silicidation was performed by RTA in $\mathrm{N}_{2}$ atmosphere. The thicknesses of Co and Fe films were measured by a quartz oscillator thickness monitor. The sample structure and a central experimental condition were as follows: the SOI thickness $d_{\text {SOI }}$ of $40 \mathrm{~nm}$, the Co thickness $d_{\mathrm{Co}}$ of $45 \mathrm{~nm}$, the Fe thickness $d_{\mathrm{Fe}}$ of $24 \mathrm{~nm}$, the annealing temperature $T_{A}$ of 
$700{ }^{\circ} \mathrm{C}$, and the annealing time $t_{A}$ of $4 \mathrm{~min}$. These parameters were used throughout following experiments, unless otherwise noted. Hereafter, $\mathrm{Co}_{2} \mathrm{FeSi}$ will be expressed as CFS even for off-stoichiometric composition.

\section{$\underline{\mathrm{CO}}_{2} \underline{\mathrm{FeGe}}$}

For the formation of $\mathrm{Co}_{2} \mathrm{FeGe}$, we prepared a pseudo-GOI substrate ${ }^{15}$ that consists of a 40-nm-thick epitaxial Ge film grown by low-temperature molecular beam epitaxy (LTMBE) on a 2-nm-thick ultra-thin SOI layer. Deposition of transition metal layers and RTA condition are the same as the case of CFS described above. Hereafter, the sample structure before the RTA treatment is referred to as $\mathrm{Fe} / \mathrm{Co} / \mathrm{GOI} / \mathrm{BOX} / \mathrm{Si}$, and $\mathrm{Co}_{2} \mathrm{FeGe}$ will be denoted by CFG.

\section{Results and discussion}

\section{Composition analyses}

Figures 2(a) and (b) show depth profiles of $\mathrm{Co}, \mathrm{Fe}, \mathrm{Si}$, and $\mathrm{O}$ in a reference sample using a $\mathrm{Si}$ substrate and in a RTA-treated $\mathrm{Fe} / \mathrm{Co} / \mathrm{SOI} / \mathrm{BOX} / \mathrm{Si}$ sample. The depth profiles were measured by secondary ion mass spectroscopy (SIMS) with the MCs + technique $^{16}$, and the SIMS intensity of each element was calibrated by Rutherford backscattering and particle induced x-ray emission measurements. When the silicidation was performed using an ordinary $\mathrm{Si}$ substrate, $\mathrm{Fe}$ and $\mathrm{Co}$ were much deeply diffused into the $\mathrm{Si}$ substrates, as shown in Fig. 2(a). For the RTA-treated Fe/Co/SOI/BOX/ Si sample, the diffusion of $\mathrm{Co}$ and $\mathrm{Fe}$ was completely blocked by the BOX layer, as expected, and the $\mathrm{Co}, \mathrm{Fe}$, and Si concentrations in the RTA-formed film exhibited the plateau profiles. The concentrations of $\mathrm{Co}, \mathrm{Fe}$, and $\mathrm{Si}$ were $48 \%, 30 \%$, and $22 \%$, respectively, which was slightly off-stoichiometric composition. The deviation from the stoichiometric composition would be caused by the uncalibrated quartz oscillator thickness monitor.

Figure 2(c) shows depth profiles for a RTA-treated $\mathrm{Fe} / \mathrm{Co} / \mathrm{GOI} / \mathrm{BOX} / \mathrm{Si}$ sample. As well as the case of CFS, the diffusion of Co and Fe are completely blocked by the BOX layer and the plateau profiles are also obtained. The chemical composition of $\mathrm{Co}, \mathrm{Fe}$ and Ge was $45 \%, 31 \%$, and $24 \%$, respectively.

\section{Crystallographic features}

The crystallographic features of the RTA-formed CFS film were characterized by XRD. Figure 3(a) shows the X-ray diffraction (XRD) pattern of the RTA-formed CFS film by $\theta-2 \theta$ measurements. After the $\mathrm{Fe} / \mathrm{Co} / \mathrm{SOI} / \mathrm{BOX} / \mathrm{Si}$ sample was annealed at $T_{A}=$ $700{ }^{\circ} \mathrm{C}$, the RTA-formed film showed strong CFS (220) and (440) diffraction peaks and no other diffraction peaks of CFS were observed in the $2 \theta$ range between $20^{\circ}$ and $120^{\circ}$, indicating that the RTA-formed CFS thin film was highly (110) oriented. The lattice constant of the CFS film was $0.5636 \mathrm{~nm}$, which was slightly smaller than that of the bulk value $\left(0.5647 \mathrm{~nm}^{17}\right)$. The deviation of the lattice constant from the bulk value would be caused by the off-stoichiometric composition. Note that when an ordinary Si substrate was used, the sample showed no CFS-related diffraction peak. These results were consistent with the above-described SIMS observations shown in Fig. 1. The rocking curve analysis of the (220) diffraction with respect to the tilt angle $\psi$ of the sample stage and its related pole figure analysis indicated that the CFS thin film was columnar poly- 
crystalline. Transmission electron microscope (TEM) observations revealed the formation of columnar grains with the grain boundaries perpendicularly penetrating through the whole film.

The crystallographic structure of the RTA-formed CFS film was evaluated from XRD superlattice measurements. Since the RTA-formed CFS film was strongly (110)-oriented, the CFS (200) and (111) diffractions indicating fully ordered $L 2_{1}$ structure can be observed by inclining the sample plane toward $45^{\circ}$ and $35.3^{\circ}$. The results are shown in Fig. 4(a). The strong-(111) and (200) superlattice diffraction imply highly $L 2_{1}$-ordered structure. The quantitative evaluation of the degree of $L 2_{1}$ order is underway.

Figure 3(b) shows the XRD $\theta-2 \theta$ pattern of the CFG film formed by RTA. The (002) and (202) diffraction peaks were simultaneously observed, indicating less orientation feature in comparison with the case of CFS. The intensity of the (002) diffraction was comparable to that of the (202) diffraction, as shown in the figure. From the ICDD (international centre for diffraction data) database, the intensity of CFS (202) should be 100 times stronger than that of CFS (002). Therefore, the predominant orientation of the RTA-formed CFG film was (001).

Figure 4(b) shows the superlattice diffraction of the CFG film. Because of the (001)orientation of the CFG film, the (202) and (111) diffractions can be observed by the tilt angles of $45^{\circ}$ and $54.7^{\circ}$ from the sample plane, respectively. The formation of $L 2_{1}$ ordered structure is confirmed from the superlattice diffractions as shown in Fig. 4(b). The weak intensity of the (111) diffraction indicats the low degree of $L 2_{1}$ order. It is necessary for the formation of CFG with the higher degree of $L 2_{1}$ order to optimize the RTA condition.

\section{Magnetic properties}

The magnetic properties of the CFS and CFG films were measured by using a superconductive quantum interference device (SQUID) magnetometer. The magnetic moment per unit cell of the CFS sample was $5.2 \mu_{\mathrm{B}}$, which is slightly smaller than the bulk value $\left(6 \mu_{\mathrm{B}}\right)^{8}$. This might be caused by the deviation of chemical composition from stoichiometric CFS, and by the incorporation of oxygen during RTA. For the CFG film, the magnetic moment was $4.8 \mu_{\mathrm{B}}$, which was also slightly smaller than the bulk value $\left(5.54 \mu_{\mathrm{B}}\right)^{10}$. This may be related to the low degree of order. Figure 5 shows the $M-T$ curves of the CFS (a solid-line) and CFG (a dashed-line) films. The Currie temperatures of the CFS and CFG films were much higher than $400 \mathrm{~K}$ (that is the highest temperature examined in this study).

A solid curve in Fig. 6(a) shows the magnetic circulardichroism (MCD) spectrum of the RTA-formed CFS film $\left(T_{A}=700{ }^{\circ} \mathrm{C}\right)$. MCD spectra for its as-deposited $\mathrm{Fe} / \mathrm{Co} / \mathrm{SOI} / \mathrm{BOX} / \mathrm{Si}$ sample, an as-deposited $\mathrm{Co} / \mathrm{SOI} / \mathrm{BOX} / \mathrm{Si}$ sample, and a RTA-treated $\mathrm{Fe} / \mathrm{Co} / \mathrm{SiO}_{2} / \mathrm{Si}$ sample with $T_{A}=700{ }^{\circ} \mathrm{C}$ are also shown in the figure as references. All the spectra were measured at room temperature with a reflection configuration with a magnetic field $H$ of $1 \mathrm{~T}$ applied perpendicular to the sample plane. In general, a MCD spectrum sensitively reflects the band structure of a material. All the reference samples showed different spectral features, as shown in Fig. 6(a). The spectral features of the RTA-formed CFS film were clearly different from those of reference samples, indicating that the formation of the $L 2_{1}$-phase full-Heusler alloy by the RTA-induced silicidation significantly modified its band structure. In addition, the MCD spectra of the RTAformed CFS film measured with the several magnitudes of the applied magnetic field exhibited identical magnetic field dependence, i.e., when the intensity of these spectra 
were normalized, these spectra were completely overlapped with one another, as shown in Fig. 6(b) and its inset. This indicates that the RTA-formed CFS film was magnetically homogeneous; in other words, ferromagnetic precipitates (residual $\mathrm{Co}, \mathrm{Fe}$, and other ferromagnetic silicides) can be excluded for the origin of the ferromagnetism in the CFS film. Figure 7 and its inset show MCD spectra of the CFG film with several perpendicular magnetic field and the normalized MCD spectra, respectively. The CFG film was also magnetically homogeneous as well as the case of the CFS film.

\section{Summary}

In summary, $L 2_{1}$-phase CFS and CFG full-Heusler alloy thin films were successfully formed by RTA-induced silicidation and germanidation, utilizing SOI and GOI substrates. This technique is fully compatible with metal-source/drain process in advanced CMOS technology and thus is promising for the fabrication of MOSFET-based spin transistors.
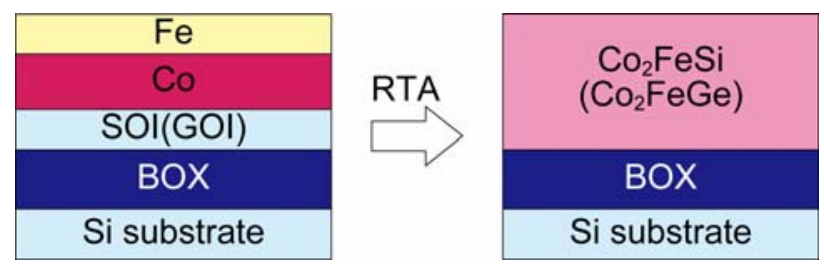

Figure 1. Schematic illustration of the proposed CFS (CFG) formation process using a SOI (GOI) substrate.

(a)

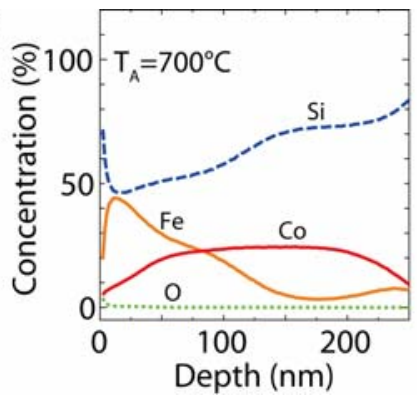

(b)

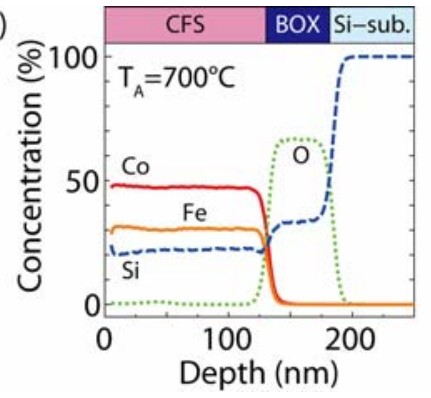

(c)

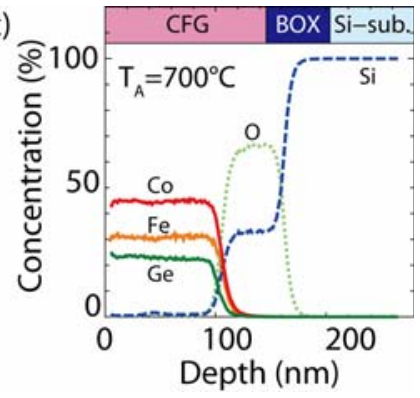

Figure 2. SIMS depth profiles of $\mathrm{Co}, \mathrm{Fe}, \mathrm{Si},(\mathrm{Ge})$, and $\mathrm{O}(\mathrm{a})$ in a RTA-treated $\mathrm{Fe} / \mathrm{Co} / \mathrm{Si}$ sample (b) in a RTA-treated $\mathrm{Fe} / \mathrm{Co} / \mathrm{SOI} / \mathrm{BOX} / \mathrm{Si}$ sample, and (c) in a RTA-treated $\mathrm{Fe} / \mathrm{Co} / \mathrm{GOI} / \mathrm{BOX} / \mathrm{Si}$ sample. 

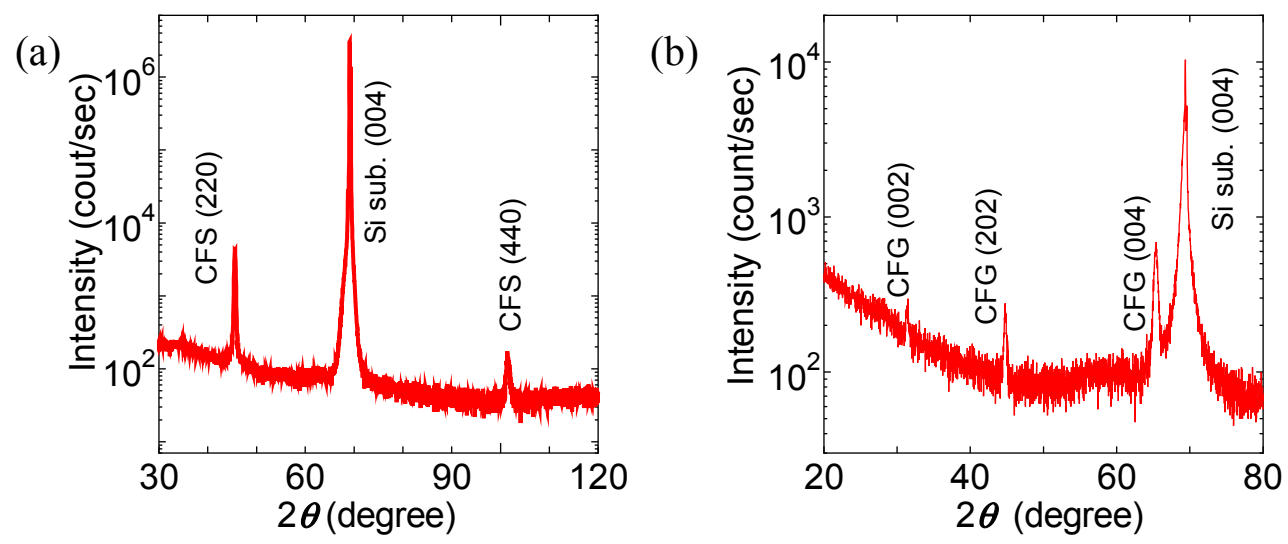

Figure 3. XRD patterns of the RTA-formed (a) CFS and (b) CFG films.
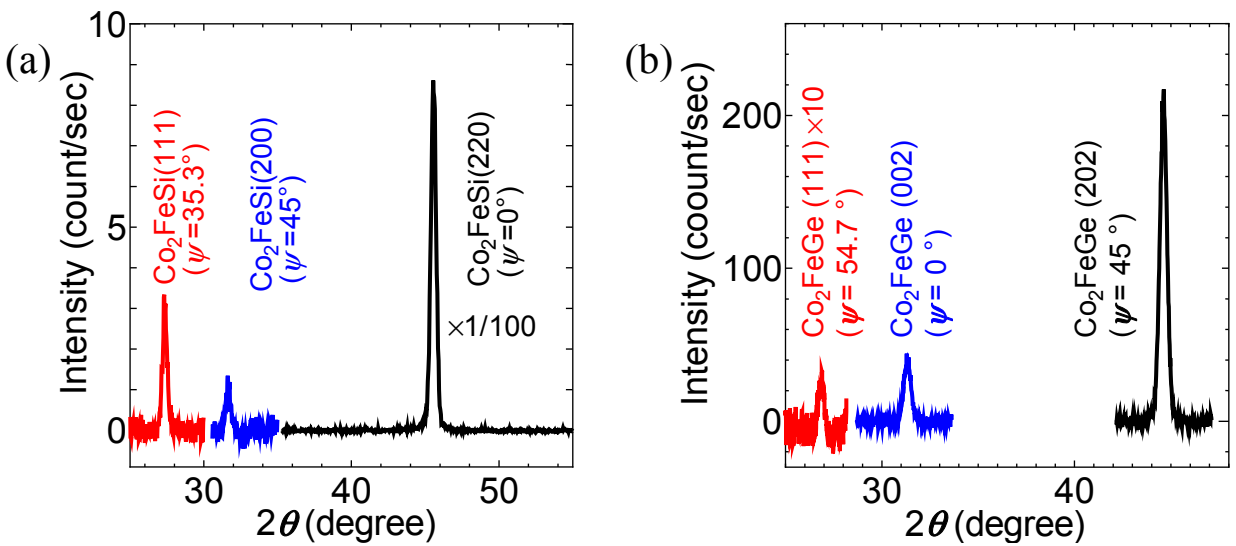

Figure 4. Superlattice diffraction of the RTA-formed (a) CFS (b) CFG films. $\psi$ is the tilt angle of the sample plane.

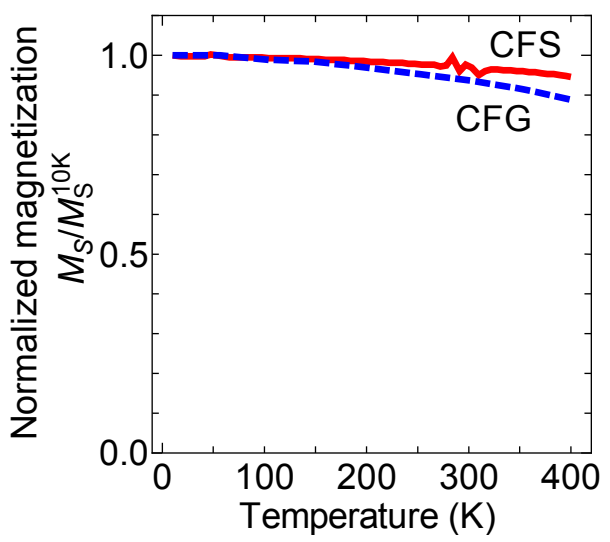

Figure 5. $M-T$ curves of the RTA-formed CFS (solid line ) and CFG (dashed line) films. 
(a)

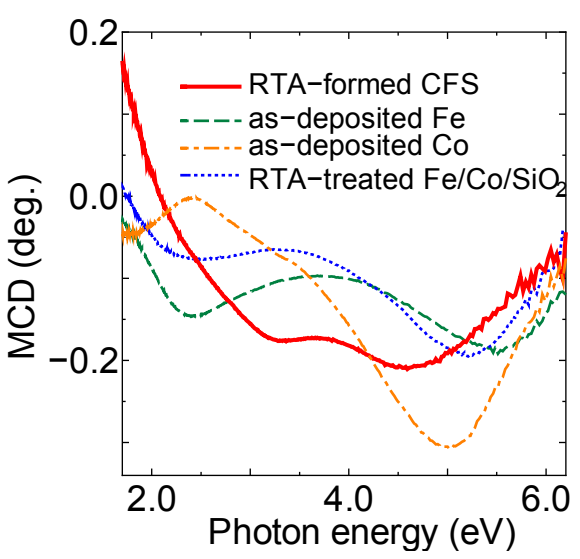

(b)

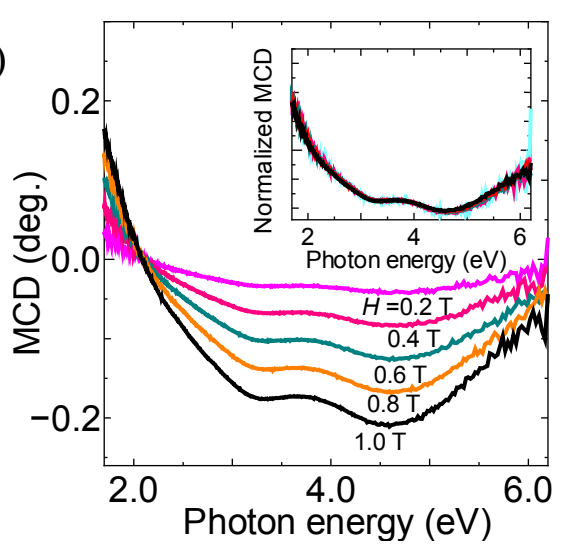

Figure 5. (a)MCD spectra of the RTA-formed CFS film (solid line) and reference samples (the others). (b) MCD spectra of the RTA-formed CFS film with several perpendicular magnetic fields. The inset shows the normalized MCD spectra.

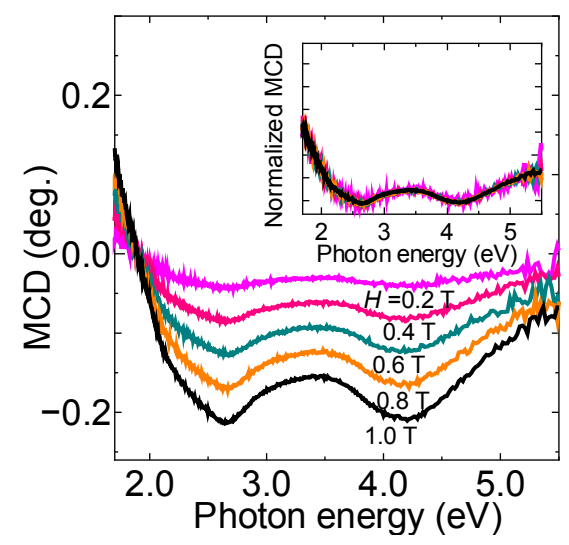

Figure 6. MCD spectra of the RTA-formed CFG film with with several perpendicular magnetic fields. The inset is the normalized MCD spectra.

\section{Acknowledgments}

The authors would like to thank Professor H. Munekata, Tokyo Institute of Technology, Professors S. Takagi and M. Tanaka, The University of Tokyo for fruitful discussions and encouragements. This work was in part supported by Grant-in-Aid for Scientific Research on Priority Area "Technology Evolution for Silicon Nano-Electronics" from MEXT and the CREST program of JST. 


\section{References}

1. S. Datta and B. Das, Appl. Phys. Lett., 56, 665 (1990)

2. M. Johnson, Science, 260, 320 (1993); IEEE Spectrum, 41, 47 (1994); IEEE Potentials, 31, 26 (1994).

3. S. Sugahara and M. Tanaka, Appl. Phys. Lett., 84, 2307 (2004)

4. S. Sugahara, IEE Proc.: Circuits Devices Syst., 152, 355 (2005).

5. R. A. de Groot, F. M. Mueller, P. G. van Engen, and K. H. J. Buschow, Phys. Rev. Lett., 50, 2024 (1983).

6. J. M. Larson and J. P. Snyder, IEEE Trans. Electron Devices, 53, 1048 (2006).

7. K. Inomata, S. Okamura, A. Miyazaki, M. Kikuchi, N. Tezuka, M. Wojcik, and E. Jedryka, J. Phys. D, 39, 816, (2006).

8. S. Wurmehl, G. H. Fecher, H. C. Kandpal, V. Ksenofontov, C. Felser, H. Lin, and J. Morais, Phys. Rev. B, 72, 184434 (2005).

9. H. Kijima, T. Ishikawa, T. Marukame, K.-I. Matsuda, T. Uemaru, and M. Yamamoto, J. Magn. Magn. Mater., 310, 2006 (2007).

10. K. H. J. Bushow, P. G. v. Engen, and R. Jongebreur, J. Magn. Magn. Mater., 38, 1 (1983).

11. T. Ishikawa, T. Marukame, K. Matsuda, T. Uemura, M. Arita, and M. Yamamoto, J. Appl. Phys. 99, 08J110 (2006).

12. T. Maeda, K. Ikeda, S. Nakaharai, T. Tezuka, N. Sugiyama, Y. Moriyama, and S. Takagi, IEEE Elec. Device Lett., 26, 102 (2005).

13. Y. Takamura, R. Nakane, H. Munekata, and S. Sugahara, J. Appl. Phys., 103, 07D719 (2008).

14. R. Nakane, M. Tanaka, and S. Sugahara, Appl. Phys. Lett., 89, 192503 (2006).

15. T. Uehara, H. Matsubara, R. Nakane, S. Sugahara, and S. Takagi, Jpn. J. Appl. Phys., 46, 2117 (2007).

16. Y. Gao, J. Appl. Phys., 64, 3760 (1988).

17. K. H. J. Buschow, P. G. van Engen, and R. Jongebreur, J. Magn. Magn. Mater., 38, 1 (1983).

18. International Centre for Diffraction Data, 04-003-7819. 